\title{
Inelastic Analysis of Circular Dampers under in-Plane Loading
}

\author{
Yen-Po Wang ${ }^{1}$, Chien-Liang Lee ${ }^{2}$, Shih-Chiao Huang ${ }^{1}$, Min-Che Tu${ }^{1}$ \\ ${ }^{1}$ Dept. Civil Engineering, National Chiao-Tung University \\ 1001 Ta-Hsueh Road, Hsinchu, Taiwan \\ ypwang@mail.nctu.edu.tw; joejoe9193.cv03g@mail.nctu.edu.tw; jimmy801015@gmail.com \\ ${ }^{2}$ School of Civil Engineering and Architecture/Xiamen University of Technology \\ No. 600 Ligong Road, Jimei District, Xiamen, 361024, Fujian Province, China \\ cllee63@hotmail.com
}

\begin{abstract}
In this paper, a methodology to determine the energy-dissipative characteristics of circular dampers under cyclic in-plane loading is proposed based on the inelastic stress analysis of curved beams with bending and shear coupling. Taking advantages of symmetry, a quarter of the circular damper in form of a curved cantilever beam with shear and bending applied simultaneously to the free end is considered in the analysis. The yield load, the corresponding yield displacement, and consequently the initial stiffness of the damper can be found analytically using the classical theory of elasticity in polar coordinate. The inelastic force-displacement relationship of the curved beam under monotonically increasing (or decreasing) load is obtained by performing the inelastic stress analysis numerically in an incremental manner. The force-displacement relationship so determined is conceived as the skeleton or backbone curve from which the hysteretic loops of the damper can be reconstructed by adopting Masing's rule. This hysteresis characterizes the mechanical behaviour of the circular damper with information of initial stiffness, yield strength, post-yielding stiffness, ductility and energy-dissipative capacity at a specified deformation. The proposed methodology may serve as an effective tool in the preliminary design stage of in-plane type metallic yielding damper to meet the desired specifications before fabrication for component test and practical use.
\end{abstract}

Keywords: inelastic stress analysis, elasticity, curved beam, total deformation theory, strain-compatibility, boundaryvalued problem, Masing's rule, Hysteretic loop

\section{Introduction}

Supplemental energy-dissipative dampers such as ADAS[1], TADAS[2] or Pre-bent stripe[3] that utilize the strength and ductility of steel plates have been widely adopted for seismic structural control in a passive manner. These metallic dampers are in common designed to deform in an out-of-plane flexural mode by resisting the loading with the weak axis of the steel plates. For the sake of material saving, it is natural to think of designing metallic dampers to deform in an in-plane flexural mode [4-7]. Primary concerns of in-plane type dampers, however, are their restricted deforming capability and early failure due to lateral instability caused by local buckling and stress concentration of the steel plates under in-plane loadings. As an effort to minimize the aforementioned limitations, modification of the existing in-plane dampers to meet the desired strength and deforming capacity is attempted before practical use becomes a reality.

Preliminary component test of a half-circular arch damper originated from its portal-frame counterpart suggests its potential in practical use with a drastic improvement in ultimate strength and ductility by reducing considerably the effect of warping and stress concentration [7]. Performance of the half-circular arch damper can be further improved by considering a closed-form circular ring in a self-balanced deforming configuration. Taking advantages of symmetry, a quarter of the circular damper in form of a curved cantilever beam with shear and bending applied simultaneously to the free end is considered in the analysis. Methodology for the stress analysis of curved beams in the elastic stage has been well developed in the theory of elasticity [8]. Inelastic stress analysis for curved beams, however, is limited to pure bending conditions. Eraslan and Arslan [9] proposed a concise analytical model for the elastic-plastic stress analysis of a strain hardening curved beam under pure bending with solutions for both plane stress and plane strain conditions according to Tresca's yielding criterion. Eraslan and Arslan [10] conducted also a computational study on the inelastic stress analysis of nonlinear hardening curve beam under pure bending in plane stress using von Mises' yielding criterion. With the 
loading restrained to pure bending, their studies provide a sound and comprehensive framework for inelastic stress analysis of curved beams with strain hardening material. In-plane lateral forces applying to a quarter of the circular dampers, however, make it a curved cantilever subjected to moment and shear simultaneously at the ends. Under such circumstances the model derived by Eraslan and Arslan[9,10] is no longer valid. To get more insight of the elastic-plastic behaviour of curved beams under bending and shear coupling, Wang et. al. [11] develop a comprehensive inelastic stress analysis model inspired by the work of Eraslan and Arslan[9,10]. Extended from the classical theory of elasticity, the proposed computational model adopts the generalized Hook's law in plane stress with consideration of the plastic strains defined by the total deformation theory. The swift-type nonlinear hardening law is considered for the inelastic constitutive relations of the material with its behaviour governed by von Mises' yield criterion. With the introduction of a certain form of Airy stress function that satisfies the compatibility equation in polar coordinates, the strain-compatibility equation with the corresponding boundary conditions can be simplified as a second-order ordinary differential equation of a properly defined generalized stress function dependent only on radius of the wide curved beam. Solution of this ODE equation becomes a boundary-valued problem that can be solved numerically in both elastic and inelastic stages. As a further extension, the displacement fields of the curved beam are derived in a unified manner, regardless of elastic or plastic stage. The inelastic force-displacement relationship of the curved beam under monotonically increasing (or decreasing) load is then obtained by performing the inelastic stress analysis step-by-step numerically in an incremental manner. The force-displacement relationship so determined is conceived as the skeleton or backbone curve from which the hysteretic loops of the damper can be reconstructed by adopting Masing's rule [12]. This hysteresis characterizes the mechanical behaviour of the circular damper with information of initial stiffness, yield strength, post-yielding stiffness, ductility and energy-dissipative capacity at a specified deformation. The proposed methodology may serve as an effective tool in the preliminary design stage of inplane type metallic yielding damper.

\section{Computational Model}

A circular damper loaded by an in-plane compressive force $2 P$ is illustrated in Fig. 1(a). A free body of the lower part of the damper can be represented by a circular arch, as shown in Fig. 1(b). Both ends of the arch are subjected to a bending moment $M=\pi l e s$ and a shear $P$ simultaneously, as obtained from elementary structural analysis. Taking advantage of symmetry with respect to the vertical axis, the analytical model can be further reduced to a quarter of the damper in terms of a curved cantilever subjected to coupling of shear and moment at its end, as indicated in Fig. 1(c).

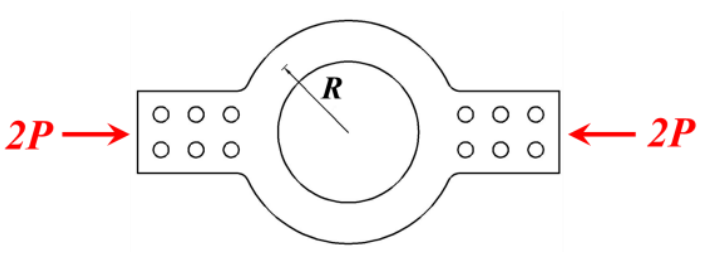

(a) in-Plane Flexural Damper

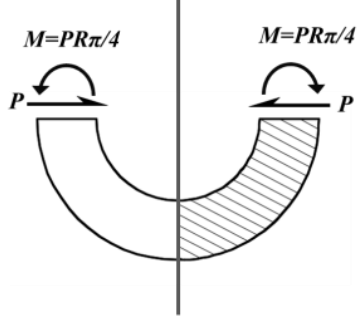

(b) Free-body of the Circular Arch

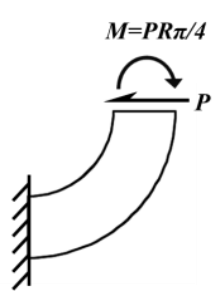

(c) Curved Cantilever

Fig. 1: The Damper and the Corresponding Analytical Model.

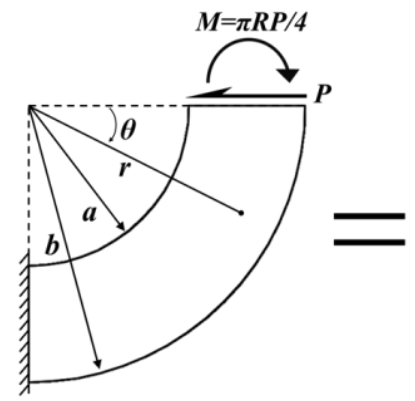

(a) P-M Coupling

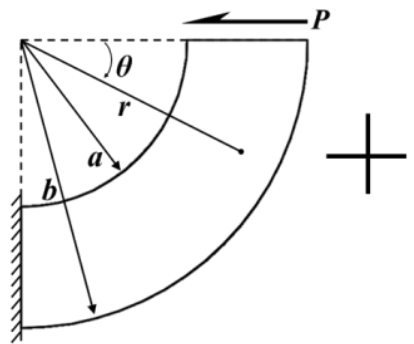

(b) End-Shear (P) only

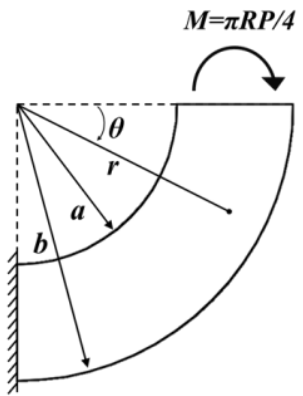

(c) Pure Bending (M)

Fig. 2: Resolving Diagram of the Loads on the Circular Cantilever. 


\subsection{Fundamental of Solid Mechanics}

The curved beam with inner radius $a$ and outer radius $b$ is considered, as shown in Fig. 2. For plane stress problems, the equilibrium equations of the curved beam with a uniform thickness $\left(t_{z}\right)$ can be expressed as [8]

$$
\begin{gathered}
\frac{\partial \sigma_{r}}{\partial r}+\frac{1}{r} \frac{\partial \tau_{r \theta}}{\partial \theta}+\frac{\sigma_{r}-\sigma_{\theta}}{r}=0 \\
\frac{\partial \tau_{r \theta}}{\partial r}+\frac{1}{r} \frac{\partial \sigma_{\theta}}{\partial \theta}+\frac{2 \tau_{r \theta}}{r}=0
\end{gathered}
$$

Where $\sigma_{r}, \sigma_{\theta}$ are respectively the normal stress in the radial and tangential directions, and $\tau_{r \theta}$ the shear stress. These stress components can be further written in terms of an Airy stress function $\varphi(r, \theta)$ as

$$
\begin{array}{cc}
\sigma_{r}=\frac{1}{r} \frac{\partial \varphi}{\partial r}+\frac{1}{r^{2}} \frac{\partial^{2} \varphi}{\partial \theta^{2}} & \sigma_{r}=\frac{1}{r} \frac{\partial \varphi}{\partial r}+\frac{1}{r^{2}} \frac{\partial^{2} \varphi}{\partial \theta^{2}} \\
\sigma_{\theta}=\frac{\partial^{2} \varphi}{\partial r^{2}} \sigma_{\theta}=\frac{\partial^{2} \varphi}{\partial r^{2}} & \sigma_{\theta}=\frac{\partial^{2} \varphi}{\partial r^{2}} \\
\tau_{r \theta}=-\frac{\partial}{\partial r}\left(\frac{1}{r} \frac{\partial \varphi}{\partial \theta}\right) & \tau_{r \theta}=-\frac{\partial}{\partial r}\left(\frac{1}{r} \frac{\partial \varphi}{\partial \theta}\right)
\end{array}
$$

Which satisfy the equilibrium equation (1). The strains are related to the displacement fields $(u, v)$ respectively for the radial and tangential displacements as

$$
\begin{gathered}
\varepsilon_{r}=\frac{\partial u}{\partial r} \\
\varepsilon_{\theta}=\frac{u}{r}+\frac{1}{r} \frac{\partial v}{\partial \theta} \\
\gamma_{r \theta}=\frac{1}{r} \frac{\partial u}{\partial \theta}+\frac{\partial v}{\partial r}-\frac{v}{r}
\end{gathered}
$$

Where $\varepsilon_{r}, \varepsilon_{\theta}$ are the normal strains in the radial and tangential direction, respectively, and $\gamma_{r \theta}$ the shear strain. The strain-compatibility equation [13] can be expressed in terms of the Airy stress function $\varphi(r, \theta)$ as

$$
\left(\frac{\partial^{2}}{\partial r^{2}}+\frac{1}{r} \frac{\partial}{\partial r}+\frac{1}{r^{2}} \frac{\partial^{2}}{\partial \theta^{2}}\right)\left(\frac{\partial^{2} \varphi}{\partial r^{2}}+\frac{1}{r} \frac{\partial \varphi}{\partial r}+\frac{1}{r^{2}} \frac{\partial^{2} \varphi}{\partial \theta^{2}}\right)=0
$$

The von Mises' yielding criterion adopted in this study is defined as

$$
\sigma_{v M}=\sqrt{\sigma_{r}^{2}-\sigma_{r} \sigma_{\theta}+\sigma_{\theta}^{2}+3 \tau_{r \theta}} \geq \sigma_{y}
$$

The material becomes partially plastic wherever the inequality holds. The generalized Hooke's law in accordance with the total deformation theory [14] is written as 


$$
\begin{gathered}
\varepsilon_{r}=\varepsilon_{r}^{P}+\frac{1}{E}\left(\sigma_{r}-v \sigma_{\theta}\right) \\
\varepsilon_{\theta}=\varepsilon_{\theta}^{P}+\frac{1}{E}\left(\sigma_{\theta}-v \sigma_{r}\right) \\
\gamma_{r \theta}=\gamma_{r \theta}^{p}+\frac{\tau_{\gamma \theta}}{G}
\end{gathered}
$$

Where the plastic strains are defined by

$$
\begin{gathered}
\varepsilon_{r}^{P}=\frac{\varepsilon_{E Q}}{\sigma_{v M}}\left(\sigma_{r}-\frac{1}{2} \sigma_{\theta}\right)=\frac{1}{E_{p}}\left(\sigma_{r}-\frac{1}{2} \sigma_{\theta}\right) \\
\varepsilon_{\theta}^{P}=\frac{\varepsilon_{E Q}}{\sigma_{v M}}\left(\sigma_{\theta}-\frac{1}{2} \sigma_{r}\right)=\frac{1}{E_{p}}\left(\sigma_{\theta}-\frac{1}{2} \sigma_{r}\right) \\
\gamma_{r \theta}^{p}=\frac{\tau_{r \theta}}{G_{p}}=\frac{3}{E_{p}} \tau_{r \theta}
\end{gathered}
$$

In which the equivalent plastic strain of a swift-type nonlinear hardening law is considered as

$$
\begin{aligned}
& \varepsilon_{E Q}=\frac{\left(\frac{\sigma_{v M}}{\sigma_{y}}\right)^{m}-1}{H} \quad \text { for } \quad \sigma_{v M} \geq \sigma_{y} \\
& =0 \quad \text { for } \sigma_{v M}<\sigma_{y}
\end{aligned}
$$

Where parameters $m$ and $H$ characterize the post-yielding behaviour of the material. The hardening parameters $m=1.2$ and $\mathrm{H}=0.25$ are considered in this study.

\subsection{Pure Bending}

Under a pure bending condition as indicated in Fig. 2(c), the radial and tangential normal stresses can be respectively expressed in terms of a generalized stress function $Y_{1}(r)$ suggested by Eraslan and Arslan[10] as

$$
\sigma_{r}=Y_{1}(r) / r
$$

and,

$$
\sigma_{\theta}=\frac{d Y_{1}(r)}{d r}
$$

Using the strain-displacement relations in eq.(3) and taking into account the fact of shear strain being zero, the strain-

compatibility eq.(4) can be written in the form of a second-order ordinary differential equation of $Y_{1}$ upon substitution of the generalized Hooke's law (6) for the strains as 


$$
r^{2} Y_{1}^{\prime \prime}+r Y_{1}^{\prime}-Y_{1}=r\left(\phi_{M}+\varepsilon_{r}^{p}-\varepsilon_{\theta}^{p}-r \frac{d \varepsilon_{\theta}^{p}}{d r}\right)
$$

Where $\phi_{M}$ is an integration constant. Eq.(11) can be rearranged in the following form as

$$
Y_{1}^{\prime \prime}=F\left(r, Y_{1}, Y_{1}^{\prime}\right)
$$

with the following boundary conditions

$$
\begin{gathered}
Y_{1}(a)=Y_{1}(b)=0 \\
\int_{a}^{b} Y_{1}^{\prime} r d r=-M / t_{z}=\pi P R / 4 t_{z}
\end{gathered}
$$

The $4^{\text {th }}$-order Runge-Kutter algorithm is adopted for solving the ODE problem iteratively along with Newton's method in the shooting process to accelerate convergence of the boundary-valued problem, as suggested by Eraslan and Arslan [10]. Once $Y_{1}$ and its derivative $Y_{1}^{\prime}$ are solved, the stress components can be found by eqs.(9) and (10) immediately.

The displacement fields $(u, v)$ can be derived from the strain-compatibility equation with the strain-displacement relations (3) and boundary conditions

$$
u\left(r, \frac{\pi}{2}\right)=0 ; v\left(r, \frac{\pi}{2}\right)=0 ; \frac{\partial v}{\partial r}\left(r, \frac{\pi}{2}\right)=0
$$

Which leads to [15]

$$
\begin{gathered}
u(r, \theta)=\left(r \varepsilon_{\theta}-r \phi_{M}\right)(1-\sin \theta) \\
v(r, \theta)=r \phi_{M} \theta-\left(r \varepsilon_{\theta}-r \phi_{M}\right) \cos \theta-\phi_{M}\left(\frac{\pi r}{2}\right)
\end{gathered}
$$

The lateral displacement of the curved beam at the free end, $u_{1}(R, 0)$, subjected to pure bending can be estimated to be

$$
u_{1}(R, 0)=R\left[\frac{1}{E_{P}}\left(Y_{1}^{\prime}-\frac{Y_{1}}{2 R}\right)+\frac{1}{E}\left(Y_{1}^{\prime}-v \frac{Y_{1}}{R}\right)-\phi_{M}\right]
$$

\subsection{End Shear}

With a shear force acting at the end of the curved beam, as indicated in Fig. 2(b), the condition becomes more complicated with the presence of shear stress and all the stress components are now dependent on both $r$ and $\theta$. However, if we loan the Airy stress function, to start with, from the classical theory of elasticity [8], namely

$$
\varphi(r, \theta)=f(r) \sin \theta
$$

and introduce a generalized stress function $Y_{2}(r)$ where 


$$
Y_{2}(r)=f^{\prime}(r)-\frac{f(r)}{r}
$$

the stress components can then be written as

$$
\begin{gathered}
\sigma_{r}=\frac{Y_{2}}{r} \sin \theta \\
\sigma_{\theta}=\left(Y_{2}^{\prime}+\frac{Y_{2}}{r}\right) \sin \theta \\
\tau_{r \theta}=\frac{-Y_{2}}{r} \cos \theta
\end{gathered}
$$

As a result, the generalized Hooke's law (6) can be modified as

$$
\begin{gathered}
\varepsilon_{r}=\varepsilon_{r}^{P}+\frac{1}{E}\left[\frac{Y_{2}}{r}-v\left(Y_{2}^{\prime}+\frac{Y_{2}}{r}\right)\right] \sin \theta \\
\varepsilon_{\theta}=\varepsilon_{\theta}^{P}+\frac{1}{E}\left[Y_{2}^{\prime}+\frac{Y_{2}}{r}-v \frac{Y_{2}}{r}\right] \sin \theta \\
\gamma_{r \theta}=\gamma_{r \theta}^{p}+\frac{1}{G}\left(-\frac{Y_{2}}{r}\right) \cos \theta
\end{gathered}
$$

Upon substitution of eq. (20) for the strains into the strain-compatibility equation (4) and integrated with respect to radius $r$, it leads to

$$
r^{2} Y_{2}^{\prime \prime}+r Y_{2}^{\prime}-4 Y_{2}=\phi_{p}+\frac{E}{\sin \theta}\left(r \frac{\partial \gamma_{r \theta}^{p}}{\partial \theta}-r^{2} \frac{\partial \varepsilon_{\theta}^{p}}{\partial r}+r \varepsilon_{r}^{p}\right)
$$

Where $\phi_{P}$ is an integration constant. Eq.(21) can be similarly rearranged as

$$
Y_{2}^{\prime \prime}=F\left(r, Y_{2}, Y_{2}^{\prime}\right)
$$

with the following boundary conditions

$$
\begin{gathered}
Y_{2}(a)=Y_{2}(b)=0 \\
\int_{a}^{b} \frac{Y_{2}}{r} d r=-\frac{P}{t_{z}}
\end{gathered}
$$

The same procedure as described in Section 2.2 can be adopted to solve the boundary-valued problem of eqs. (22)-(23). Once $Y_{2}$ and its derivative $Y_{2}^{\prime}$ are solved, the stress components can be found by eqs.(19) immediately for a given crosssection at $\theta$.

The displacement fields $(u, v)$ can be derived from the strain-compatibility equation with the strain-displacement relations (3) and boundary conditions (14) to be [15] 


$$
\begin{gathered}
u(r, \theta)=\frac{\phi_{P}}{4 E}[-\pi \cos \theta+\sin \theta+(2 \theta-\sin 2 \theta) \cos \theta+\cos 2 \theta \sin \theta] \\
v(r, \theta)=-\frac{1}{E_{P}}\left(r Y_{2}^{\prime}+\frac{Y_{2}}{2}\right) \cos \theta-\frac{1}{E}\left(r Y_{2}^{\prime}+Y_{2}-v Y_{2}\right) \cos \theta-\frac{\phi_{P}}{4 E}(-\pi \sin \theta-2 \cos \theta+2 \theta \sin \theta)
\end{gathered}
$$

The lateral displacement of the curved beam at the free end, $u_{2}(R, 0)$, subjected to end shear can be estimated to be

$$
u_{2}(R, 0)=-\frac{\pi \phi_{P}}{4 E}
$$

\subsection{Bending and Shear Coupling}

If the curved beam is subjected to the coupling of bending $(M)$ and shear $(P)$ at the end simultaneously, the stresses can be obtained by summing the results from pure bending and end shear derived independently as

$$
\begin{gathered}
\sigma_{r}=\sigma_{r, 1}+\sigma_{r, 2} \\
\sigma_{\theta}=\sigma_{\theta, 1}+\sigma_{\theta, 2} \\
\tau_{r \theta}=\tau_{r \theta, 2}
\end{gathered}
$$

Where the subscript 1 denotes those obtained from pure bending and subscript 2 from end shear. Note that under the coupling loading condition, the resultant stresses from eqs. (26) should be used in checking the yielding condition via the von Mises criterion (5).

The lateral displacement of the circular damper due to the compressive load $2 \mathrm{P}$ is therefore estimated to be

$$
u(R, 0)=2\left[u_{1}(R, 0)+u_{2}(R, 0)\right]
$$

The inelastic force-displacement relation ( $2 \mathrm{P}$ vs. $u(R, 0))$ of the circular damper under monotonically increasing (or decreasing) load is obtained by performing the inelastic stress analysis numerically in an incremental manner using the proposed method. The force-displacement relationship so determined is conceived as the skeleton or backbone curve from which the hysteretic loops of the damper can be reconstructed by adopting Masing's rule [15]. This hysteresis characterizes the mechanical behaviour of the circular damper with information of initial stiffness, yield strength, post-yielding stiffness, ductility and energy-dissipative capacity (area enclosed by the hysteresis) at a desired deformation.

\section{Numerical Verification}

As an effort to demonstrate the proposed methodology, a numerical example of the inelastic stress analysis has been conducted using the circular damper shown in Fig. 1(a) with inner radius $a=120 \mathrm{~mm}$ and outer radius $b=190 \mathrm{~mm}$. Thickness of the steel plate is $28 \mathrm{~mm}$. Material properties of the steel are summarized in the following: Young's modulus $E=200 \mathrm{GPa}$, Poisson's ratio $v=0.3$ and yield stress $\sigma_{y}=325 \mathrm{MPa}$. An in-plane compressive force $2 P$ is acting inwards at the middle of the damper as illustrated in Fig. 1(a) and produces shear $P$ and bending $M=\pi R P / 4$ simultaneously on the free body indicated in Fig. 2(a). The yield strength $2 P_{y}=49.2 \mathrm{kN}$ of the damper is determined by setting the normal stress $\sigma_{\theta}(b, \pi / 2)=\sigma_{y}$ where initial yielding takes place. The inelastic force-displacement relation $(2 \mathrm{P}$ vs. $u(R, 0))$ of the circular damper under monotonically increasing (or decreasing) load is obtained by performing the inelastic stress analysis numerically in an incremental manner with $P_{i+1}=P_{i}+0.0001$ increasingly or $P_{i+1}=P_{i}-0.0001$ decreasingly step-by-step until the lateral displacement $u(R, 0)$ at the loading point reaches $\pm 40 \mathrm{~mm}$. The curve so determined as the solid line shown in Fig. 3 is called a backbone curve which exhibits the characteristics of the damper. The hysteresis of the damper (dotted 
lines) is then constructed using Masing's rule [15]. The initial stiffness $K_{1}$ reads $1222.26 \mathrm{kN} / \mathrm{mm}$ and the post-yielding stiffness $K_{2}$ reads $10.87 \mathrm{kN} / \mathrm{mm}$ from the backbone curve, as indicated in Fig. 3. The initial stiffness is comparably close to the analytical one $1230 \mathrm{kN} / \mathrm{mm}$ estimated by classical theory of elasticity. The proposed methodology may serve as an effective tool in the preliminary design stage of in-plane type metallic yielding damper to meet the desired specifications before fabrication for component test and practical use.

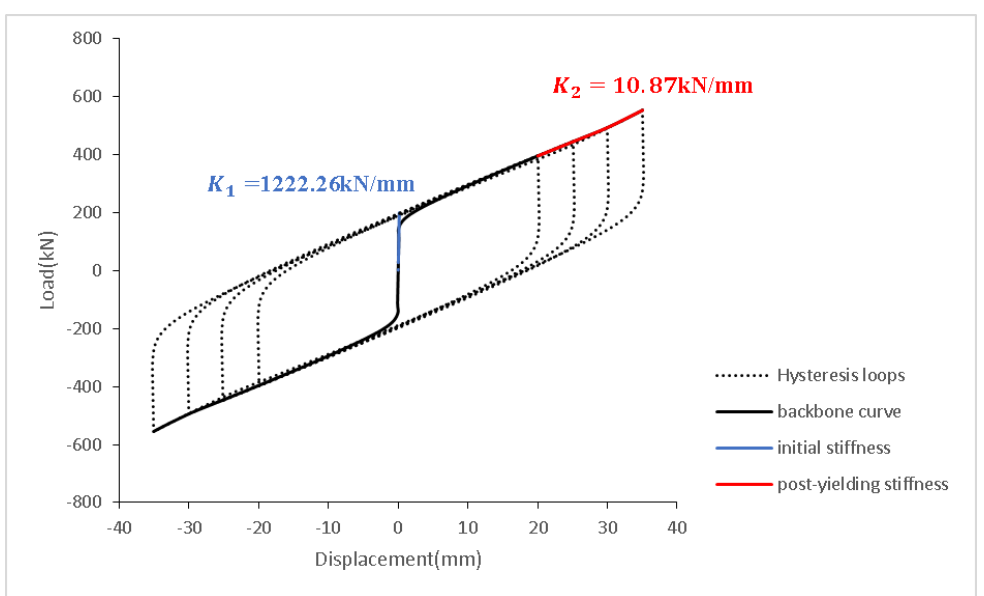

Fig. 3: Hysteresis reconstructed from the backbone curve of the damper.

\section{Conclusion}

In this paper, a methodology to determine the energy-dissipative characteristics of circular dampers under cyclic inplane loading is proposed based on the inelastic stress analysis of curved beams with bending and shear coupling developed earlier. The inelastic force-displacement relationship of the curved beam under monotonically increasing (or decreasing) load is obtained by performing the inelastic stress analysis numerically in an incremental manner. The forcedisplacement relationship so determined is conceived as the backbone curve from which the hysteretic loops of the damper can be reconstructed by adopting Masing's rule. This hysteresis characterizes the mechanical behaviour of the circular damper with information of post-yielding stiffness, ductility and energy-dissipative capacity at a desired deformation. The proposed methodology may serve as an effective tool in the preliminary design stage of in-plane type metallic yielding damper to meet the desired specifications before fabrication for component test and practical use.

\section{Acknowledgements}

This research work is granted by the Ministry of Science and Technology of Republic of China under contract MOST 104-2221-E-009-197.

\section{References}

[1] A. S. Whittaker, V. V. Bertero, C. L. Thompson, L. J. Alonso,'Seismic Testing of Steel Plate Energy Dissipation Devices," Earthquake Spectra, vol. 7, no. 4, pp. 563-604, 1991.

[2] K. C. Tsai, H. W. Chen, C. P. Hong, Y. F. Su, "Design of Steel Triangular Plate Energy Absorbers for SeismicResistant Construction," Earthquake Spectra; vol. 9, no. 3, pp. 505-528, 1993.

[3] Y. P. Wang, C. S. Chang Chien, "A Study on Using Pre-Bent Steel Strips as Seismic Energy-Dissipative Devices," Earthquake Engineering \& Structural Dynamics, vol. 38, pp. 1009-1026, 2009.

[4] W. K. Chan, F. Albermani, "Experimental Study of Steel Slit Damper for Passive Energy Dissipation," Engineering Structures, vol. 30, pp. 1058-1066, 2008.

[5] J. H. Park, K. H. Lee, "Cyclic Loading Tests of Steel Dampers Utilizing Flexure-Analogy Deformation," in Proceedings of the 15 $5^{T H}$ World Conference on Earthquake Engineering, Lisbon, Portugal, paper no. 1228, 2012.

[6] Z. Guan, J. Li, Y.Xu, "Performance Test of Energy Dissipation Bearing and Its Application in Seismic Control of a Long-Span Bridge," Journal of Bridge Engineering, ASCE, vol. 15, pp. 622-630, 2010. 
[7] Y. P. Wang, D. H. Chen, C. L. Lee, "An Experimental Study on in-Plane Arch-shaped Flexural Damper," Implementing Innovative Ideas in Structural Engineering and Project Management, Proceedings of ISEC-8, Parramatta, Australia, pp. 293-298, 2015.

[8] S. P. Timoshenko, J. N. Goodier, Theory of Elasticity, Third edition, New York, McGraw-Hill, 1970.

[9] A. N. Eraslan, E. Arslan, "A Concise Analytical Treatment of Elastic-Plastic Bending of a Strain Hardening Curved Beam,” ZAMM, vol. 88, no. 8, pp. 600-616, 2008.

[10] A. N. Eraslan, E. Arslan, "A Computational Study on the Nonlinear Hardening Curved Beam Problem," International J. Pure and Applied Mathematics, vol. 43, no. 1, pp. 129-143, 2008.

[11] Y. P. Wang., C. L. Lee, S. C. Huang, "Inelastic Stress Analysis of Curved Beams with Bending and Shear Coupling," Proceedings of the World Congress on Civil, Structural, and Environmental Engineering (CSEE'16), Prague, Czech Republic, Paper No. ICSENM 107, 2016.

[12] G. Masing, "Eigenspannungen and verfestigung beim messing," Proceedings of the 2nd International Congress of Applied Mechanics, Orell Füssliverlag, Zürich, pp. 332-335, 1926.

[13] A. P. Boresi, K. P. Chong, J. D. Lee, Elasticity in Engineering Mechanics, Third edition, Hoboken, New Jersy, John Wiley and Sons, Inc., 2011.

[14] R. M. Jones, Deformation Theory of Elasticity, Blacksburg, Virginia, Bull Ridge Publishing, 2009.

[15] S. C. Huang, "Inelastic Stress Analysis of Curved Beams under Bending and Shear Coupling," M.S. Thesis of the Department of Civil Engineering, College of Engineering, National Chiao-Tung University, Taiwan, 2016. (in Chinese) 